\title{
Second Harmonic Generating (SHG) Nanoprobes: a New Tool for Biomedical Imaging
}

\author{
Periklis Pantazis $^{1, *}$, Ye $\mathbf{P u}^{2,3}$, Demetri Psaltis ${ }^{2,3}$ and Scott Fraser ${ }^{1}$ \\ ${ }^{1}$ Beckman Institute and Division of Biology, MC 139-74, California Institute of \\ Technology, Pasadena, CA 91125, USA \\ ${ }^{2}$ Department of Electrical Engineering, MC 136-93, California Institute of Technology, \\ Pasadena, CA 91125, USA \\ ${ }^{3}$ École Polytechnique Fédérale de Lausanne, Laboratoire d'optique, STO IOA LO, BM \\ 4102 (Bâtiment BM), Station 17, CH-1015 Lausanne, Switzerland
}

\begin{abstract}
Fluorescence microscopy has profoundly changed how cell and molecular biology is studied in almost every aspect. However, the need of characterizing biological targets is largely unmet due to deficiencies associated with the use of fluorescent agents. Dye bleaching, dye signal saturation, blinking, and tissue autofluorescence can severely limit the signal-to-noise ratio (SNR). Given the photophysical properties are fundamentally different to the fluorescent agents currently used in biomedical research, second harmonic generating ( $\mathrm{SHG}$ ) nanoprobes can be suitable for biomedical imaging and can eliminate most of the drawbacks encountered in classical fluorescence systems.
\end{abstract}

Keywords: bleaching, blinking, saturation, autofluorescence, fluorescent probes

\section{INTRODUCTION}

\subsection{Fluorescent probes}

A large variety of genetically encoded fluorescent tags (reviewed in (1)) are commonly used in in vivo optical imaging since the cloning of the green fluorescent protein (GFP) from the jellyfish Aequorea victoria (2). They provide important tools for biomedical research, as they are relatively bright, photostable, color selective, and $\mathrm{pH}$ insensitive. Over the years, they have proven to be particularly important in analyzing a variety of biological processes such as gene expression, the localization and dynamics of fluorescent-tagged proteins or fluorescent marked cell populations (reviewed in $(3,4)$ ).

*pantazis@caltech.edu; phone: +1-626-395-2863; fax: +1-626-449-8599

Multiphoton Microscopy in the Biomedical Sciences IX, edited by Ammasi Periasamy, Peter T. C. So, Proc. of SPIE Vol. 7183, 71831P · (C) 2009 SPIE · CCC code: 1605-7422/09/\$18 · doi: 10.1117/12.808434 


\subsection{Limitations of Fluorescent Probes}

Yet these increasingly used tags still present numerous disadvantages. For example, fluorescent probes face two major limitations that have a significant impact on the signal strength: 1) dye saturation, because the number of photons emitted by the fluorophore in a given time is restricted by the excited state lifetime, which arises from that fact that a single fluorescent protein can only give off one or two photons in each 10 nanosecond period, independent of how bright the laser excitation, and 2) dye bleaching, which limits the total number of photons per dye. Both limit the timescale of the biological target process to be studied. In particular, directly tracking the lineage of distinct cell populations in tissue or monitoring the dynamics of molecules within single cells depend critically on long-term photostability. In addition, autofluorescence from tissue organic components due to random illumination absorption can severely limit the signal-tonoise ratio (5). Many developments tried to overcome these problems by using robust and bright organic dyes (reviewed in (6)) or semiconductor nanocrystals, quantum dots (QDs) $(7,8)$. However, luminescence signals of QDs show a stochastic, discrete on and off behavior (9-11). This intrinsic fluorescence intermittency of QDs is a major limitation (although some might argue that it provides a criterion to identify single QDs) as it creates dark windows where information is unavailable, preventing continuous tracking of individual molecule trajectories. This behavior represents a key complication for the data analysis of single particle studies, requiring specific algorithms for data analysis (12).

\section{SECOND HARMONIC GENERATING (SHG) MICROSCOPY}

\subsection{Background}

Shortly after the demonstration of the first working laser in 1960 (13), the field of nonlinear optics was introduced by the observation of SHG in a quartz crystal (14). SHG is a second-order nonlinear optical process in which two photons interacting with non-centrosymmetrical media (i.e. material lacking a generalized mirror symmetry) are combined to form a new photon with twice the energy, and therefore twice the frequency of the initial photons (Fig. 1) $(15,16)$.
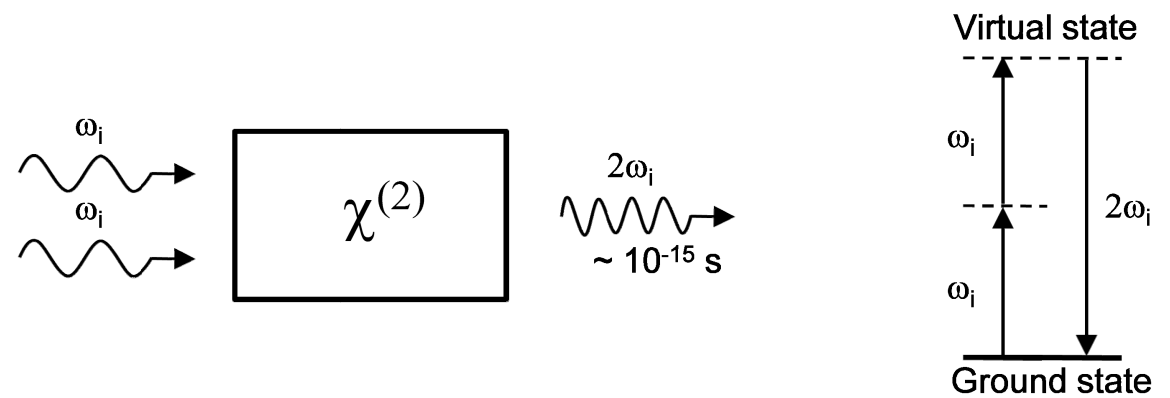

Figure 1:

(Left) When intense light is shone on materials that do not possess an inversion symmetry, the vibrating electric field of the incident beam results in the polarization of the medium, re-emitting light at the original frequency $\omega_{i}$ but also at the frequency $2 \omega_{i}$ that is twice the original one (with half of the wavelength). (Right) Unlike two-photon excited fluorescence, all of the incident radiation power at frequency $\omega_{i}$ is converted in the process of SHG to radiation at the SHG frequency $2 \omega_{i}$. And whereas two-photon excited fluorescence involves real energy transition of electrons, SHG involves only virtual energy transition. As a result, using ultrafast (femtosecond) pulsed lasers the response time of SHG is at the femtosecond level, about several orders of magnitude faster than the nanosecond response time of fluorescence, allowing very fast and sensitive detection. 
The optical response of the media can be described by expressing the induced polarization $P(\omega)$ as a power series of the optical field strength $E(\omega)$ of the incident light:

$$
P(\omega)=\chi^{(1)} \cdot E(\omega)+\chi^{(2)} \cdot E(\omega)^{2}+\chi^{(3)} \cdot E(\omega)^{3}+\ldots
$$

The coefficient $\chi^{(n)}$ is the nth-order susceptibility of the material, $\chi^{(1)}, \chi^{(2)}$, and $\chi^{(3)}$ corresponding to optical effects such as absorption or reflection, second harmonic generation, and third harmonic generation, respectively.

\subsection{Using SHG Microscopy}

Historically, SHG has been characterized extensively in bulk crystalline material. The use of SHG has grown continuously since its beginning: Second harmonic generation (SHG) microscopy is an emerging non-invasive microscopic technique for a wide range of biological and medical imaging. Given the symmetry constraints, SHG can be mainly produced from structures without inversion symmetry combined with a high degree of organization and orientation, such as anisotropic crystals or endogenous structure protein arrays in tissue. Using SHG microscopy, a variety of tissue structures have recently been imaged by virtue of intrinsic signal generated by structured proteins such as collagen fibrils in connective tissues or the actomyosin lattice of muscle cells (Fig. 2) (17).

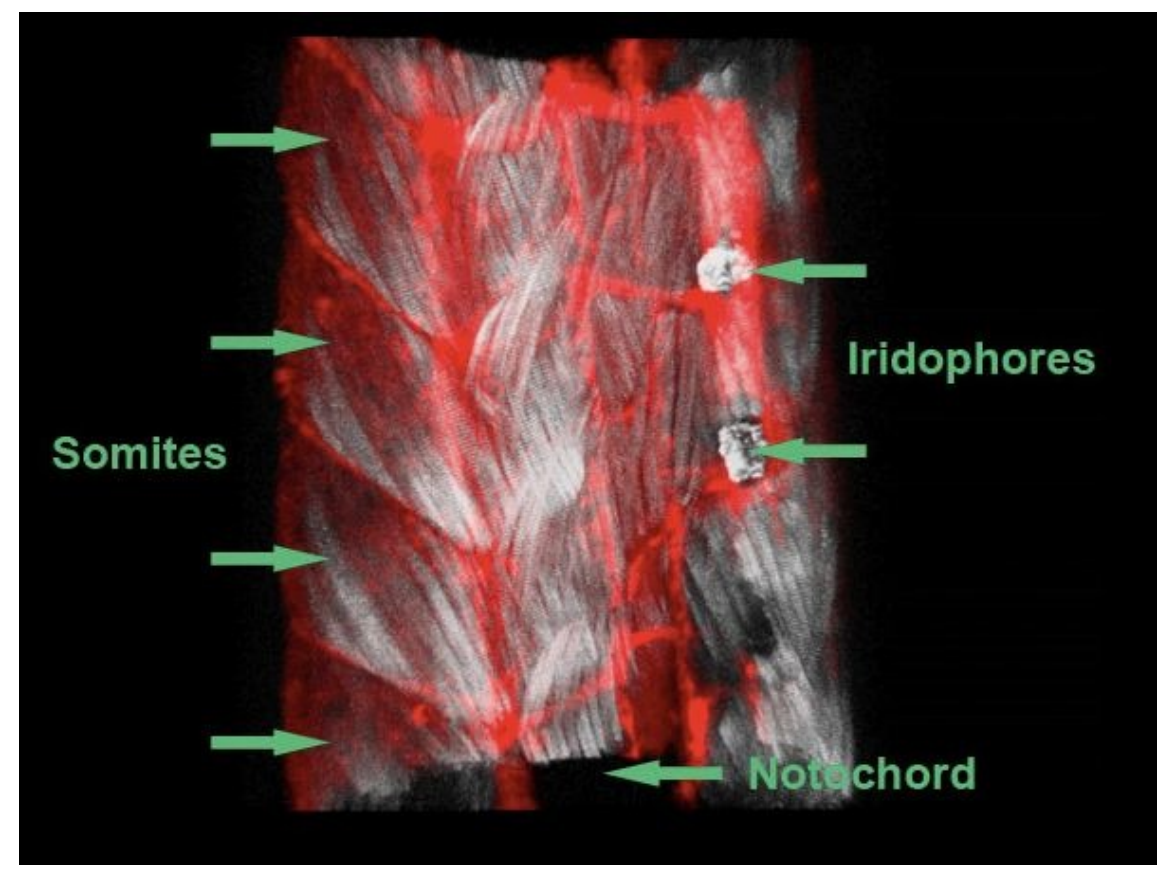

Figure 2:

SH signal of a representative $4 \mathrm{dpf}$ (day post fertilization) zebrafish tail. Signal shown in white is endogenous SHG, while red signal is a BODIPY counterstain. The two circular white clusters indicated on the right are iridophores, and the segments outlined in red along the left side of the tail are distinct somites. Within the somites, repeated white strands form fast and slow muscle patterns.

In addition, cell membranes have been labeled with asymmetric styryl dyes generating an SH signal that is sensitive to membrane potential $(18,19)$. In clinical research, the effect of molecular therapeutics on tumor growth has been successfully evaluated using non-invasive SHG imaging to estimate collagen tumor dynamics after pharmacologic intervention (20). 


\subsection{Limitations of SHG Microscopy}

However, imaging of endogenous SH signal requires high illumination intensities compared to fluorescent dyes, which can have its own phototoxic effects in long-term imaging applications. Styryl dyes present their own limitations, as high concentrations are necessary to acquire a detectable SH signal, which can affect the fluidity of membranes and may be toxic. In these SHG imaging applications, weak forwarddirected SH signal is detected, requiring microscope modifications to optimize the collection and detection of signal. In addition, the light has to pass through the specimen, making it more difficult to separate the membrane/styryl dye SH signal from the background coming from SHG of tissue structures.

\section{SHG NANOPROBES}

As a nonlinear optical process, SHG shares many features of two-photon excited fluorescence. Using pulsed lasers in the infrared wavelength range it benefits from relatively deep optical penetration and reduced ability to cause potential nonspecific phototoxicity (21). These properties are of vital importance for long time-lapse imaging. However, whereas two-photon excited fluorescence relies on nonlinear absorption, SHG is a coherent process involving only virtual energy transitions. As a result, photodamage considerations do not intrinsically exist, since SHG does not arise from an absorptive process. Since SHG is a coherent process three-dimensional molecular orientation with high spatial resolution can be observed. Moreover, the response time of SHG is at the femtosecond level, four to five orders of magnitude faster than the nanosecond response time of fluorescence, allowing very fast and sensitive detection.

SHG imaging has been used previously, but this use has been limited to special conditions, such as cultured cells, the observation of intrinsic SHG, or the collection of SHG in trans-detection. Each of these previous demonstrations is significant, but none carry the use of SHG forward to the point that it can be used in intact whole organisms.

Based on the photophysical behavior of SHG that is fundamentally different to other fluorescent agents currently used to characterize biological targets, SHG nanoprobes have the potential to provide a unique combination of advantageous properties inherent to SHG that can address the challenges imposed by fluorescent probes allowing experiments characterizing molecular targets of indefinite length, with fast acquisition rates and superb signal-to-noise ratio. They should not be limited by the significant fluorescence lifetime of the dye molecules, nor limited by the significant bleaching of fluorescence dyes (or blinking of QDs). Thus, they can offer a window into time regimes of molecular targets to which other techniques are blind. Since SHG involves only virtual energy transitions, they can provide an excellent sensitivity to smaller numbers of molecule because SHG nanoprobes should not saturate at high illumination. SHG nanoprobes can be imaged using microscope settings very similar to those required for fluorescent probe imaging, as a multi-directional SHG signal has been recently suggested for similar small particles $(22,23)$. And since SHG is a coherent process, the range of potential use of SHG nanoprobes can be much larger, as it also encompasses three-dimensional molecular orientation analysis with high spatial resolution (24). Thus, SHG nanoprobes have great potential to give insight into the dynamics of various biological targets at the molecular level with unmatched sensitivity and temporal resolution (Pantazis et al., in preparation).

\section{REFERENCES}

1. N. C. Shaner, P. A. Steinbach and R. Y. Tsien, "A guide to choosing fluorescent proteins," Nat Methods 2(12), 905-909 (2005)

2. D. C. Prasher, V. K. Eckenrode, W. W. Ward, F. G. Prendergast and M. J. Cormier, "Primary structure of the Aequorea victoria green-fluorescent protein," Gene 111(2), 229-233 (1992)

3. J. Lippincott-Schwartz, E. Snapp and A. Kenworthy, "Studying protein dynamics in living cells," Nat Rev Mol Cell Biol 2(6), 444-456 (2001) 
4. A. K. Hadjantonakis, M. E. Dickinson, S. E. Fraser and V. E. Papaioannou, "Technicolour transgenics: imaging tools for functional genomics in the mouse," Nat Rev Genet 4(8), 613-625 (2003)

5. N. Billinton and A. W. Knight, "Seeing the wood through the trees: a review of techniques for distinguishing green fluorescent protein from endogenous autofluorescence," Anal Biochem 291(2), 175$197(2001)$

6. B. N. Giepmans, S. R. Adams, M. H. Ellisman and R. Y. Tsien, "The fluorescent toolbox for assessing protein location and function," Science 312(5771), 217-224 (2006)

7. M. Bruchez, Jr., M. Moronne, P. Gin, S. Weiss and A. P. Alivisatos, "Semiconductor nanocrystals as fluorescent biological labels," Science 281(5385), 2013-2016 (1998)

$8 . \quad$ W. C. Chan and S. Nie, "Quantum dot bioconjugates for ultrasensitive nonisotopic detection," Science 281(5385), 2016-2018 (1998)

$9 . \quad$ M. Nirmal, B. O. Dabbousi, M. G. Bawendi, J. J. Macklin, J. K. Trautman, T. D. Harris and L. E. Brus, "Fluorescence intermittency in single cadmium selenide nanocrystals," Nature 383(6603), 802-804 (1996)

10. T. Basche, "Fluorescence intensity fluctuations of single atoms, molecules and nanoparticles," Journal of Luminescence 76-77(263-269 (1998)

11. S. A. Empedocles, "Influence of spectral diffusion on the line shapes of single CdSe nanocrystallite quantum dots," Journal of Physical Chemistry B 103(11), 1826 (1999)

12. M. Dahan, S. Levi, C. Luccardini, P. Rostaing, B. Riveau and A. Triller, "Diffusion dynamics of glycine receptors revealed by single-quantum dot tracking," Science 302(5644), 442-445 (2003)

13. T. H. Maiman, "Stimulated Optical Radiation in Ruby," Nature 187(4736), 493-494 (1960)

14. P. A. Franken, A. E. Hill, C. W. Peters and G. Weinreich, "Generation of Optical Harmonics," Physical Review Letters 7(4), 118 (1961)

15. R. W. Boyd, Nonlinear optics, Academic Press, San Diego, CA (2003).

16. R. L. Sutherland, D. G. McLean and S. Kirkpatrick, Handbook of nonlinear optics, Marcel Dekker, New York (2003).

17. P. J. Campagnola and L. M. Loew, "Second-harmonic imaging microscopy for visualizing biomolecular arrays in cells, tissues and organisms," Nat Biotechnol 21(11), 1356-1360 (2003)

18. M. Nuriya, J. Jiang, B. Nemet, K. B. Eisenthal and R. Yuste, "Imaging membrane potential in dendritic spines," Proc Natl Acad Sci U S A 103(3), 786-790 (2006)

19. L. Sacconi, D. A. Dombeck and W. W. Webb, "Overcoming photodamage in second-harmonic generation microscopy: real-time optical recording of neuronal action potentials," Proc Natl Acad Sci U S A 103(9), 3124-3129 (2006)

20. E. Brown, T. McKee, E. diTomaso, A. Pluen, B. Seed, Y. Boucher and R. K. Jain, "Dynamic imaging of collagen and its modulation in tumors in vivo using second-harmonic generation," Nat Med 9(6), 796-800 (2003)

21. P. Pantazis and M. Gonzalez-Gaitan, "Localized multiphoton photoactivation of paGFP in Drosophila wing imaginal discs," J Biomed Opt 12(4), 044004 (2007)

22. Y. Pavlyukh and W. Hübner, "Nonlinear Mie scattering from spherical particles," Physical Review B 70(24), 245434 (2004)

23. J. Shan, J. I. Dadap, I. Stiopkin, G. A. Reider and T. F. Heinz, "Experimental study of optical second-harmonic scattering from spherical nanoparticles," Physical Review A (Atomic, Molecular, and Optical Physics) 73(2), 023819-023814 (2006)

24. Y. Pu, M. Centurion and D. Psaltis, "Harmonic holography: a new holographic principle," Appl Opt 47(4), A103-110 (2008) 